\title{
Moving least square - one dimensional integrated radial basis function networks for time dependent problems
}

\author{
D. Ngo-Cong ${ }^{1,2}$, N. Mai-Duy ${ }^{1}$, W. Karunasena ${ }^{2} \&$ T. Tran-Cong ${ }^{1}$ \\ ${ }^{1}$ Computational Engineering \& Science Research Centre, University of \\ Southern Queensland, Australia \\ ${ }^{2}$ Centre of Excellence in Engineered Fibre Composites, University of \\ Southern Queensland, Australia
}

\begin{abstract}
This paper presents a new numerical procedure for time-dependent problems. The partition of unity method is employed to incorporate the moving least square and one-dimensional integrated radial basis function networks (MLS-1D-IRBFN) techniques in an approach that produces a very sparse system matrix and offers as a high order of accuracy as that of global 1D-IRBFN method. Moreover, the proposed approach possesses the Kronecker- $\delta$ property which helps impose the essential boundary condition in an exact manner. Spatial derivatives are discretised using Cartesian grids and MLS-1D-IRBFN, whereas temporal derivatives are discretised using high-order time-stepping schemes, namely standard $\theta$ and fourth-order Runge-Kutta methods. Several numerical examples including twodimensional diffusion equation, one-dimensional advection-diffusion equation and forced vibration of a beam are considered. Numerical results show that the current methods are highly accurate and efficient in comparison with other published results available in the literature.

Keywords: time-dependent problems, integrated radial basis functions, moving least square, partition of unity, Cartesian grids.
\end{abstract}

\section{Introduction}

In 1990, Kansa proposed a collocation scheme based on multiquadric (MQ) radial basis functions $(\mathrm{RBF})$ for the numerical solution of partial differential equations 
(PDEs) [1]. Their numerical results showed that MQ scheme yielded an excellent interpolation and partial derivative estimates for a variety of two-dimensional functions over both gridded and scattered data. The main drawback of the RBF based method is the lack of mathematical theories for the choice of the RBF shape parameter. Since this original work, many meshfree methods based on RBF have been proposed. Sharan et al. [2] employed the multiquadric approximation scheme for solution of elliptic partial differential equations using the data points in arbitrary locations with an arbitrary ordering. Zerroukat et al. [3] proposed explicit and implicit meshless methods based on global RBFs for linear advectiondiffusion-type partial differential equations on arbitrary collocation points system.

In recent years, a different approach for solving PDEs is the so-called Cartesian grid method where the governing equations are discretised on a Cartesian grid which does not conform to the immersed boundaries. This significantly reduces the grid generation cost and has a great potential over the conventional bodyfitted methods when solving problems with moving boundaries and complicated geometry. Ye et al. [4] developed a finite-volume based Cartesian grid method for simulating two-dimensional unsteady, viscous, incompressible flows with complex immersed boundaries. In their method, the immersed boundary is represented by a series of piecewise linear segments. Based on these segments, the control volume near the immersed boundary is reformed into a body-fitted trapezoidal shape. A one-dimensional integrated radial basis function networks (1D-IRBFN) collocation method for the solution of second- and fourth-order PDEs was presented by Mai-Duy and Tanner [5]. Along grid lines, 1D-IRBFN are constructed to satisfy the governing differential equations with boundary conditions in an exact manner. The 1D-IRBFN method enjoys spectral accuracy and exponential convergence for certain problems. In this method, the Cartesian grids were used to discretise both rectangular and non-rectangular problem domains. The computational cost used for the Cartesian grid generation is negligible in comparison with that required for the body-fitted mesh. Le-Cao et al. [6] presented a numerical collocation procedure based on Cartesian grids and 1D-IRBFN for simulation of natural convection defined in 2D multiply connected domains and governed by a stream function-vorticity-temperature formulation. Ngo-Cong et al. [7] extended this method to investigate free vibration of composite laminated plates based on first-order shear deformation theory. The present paper deals with the development of MLS-1D-IRBFN methods based on two frameworks of semi-discrete and fully discrete schemes for solving several numerical examples including diffusion equation, advection diffusion equation and forced vibration of a beam. In the semi-discrete scheme, the first stage is the spatial discretisation in which the spatial derivatives of the PDEs are discretised to obtain a system of ordinary differential equations (ODEs). The system is then advanced in time using ODE solvers (e.g. fourth-order Runge-Kutta scheme) to obtain the unknowns for each time step. In the fully discrete scheme, the time derivatives of the PDEs are first discretised using high-order time-stepping schemes (standard $\theta$-scheme) to obtain a sequence of steady problems. The spatial discretisation is then performed to obtain a full discretisation which is in the form of a system of algebraic 
equations. In the following, Section 2 presents the MLS-1D-IRBFN methods. Several numerical examples are then used to demonstrate the performance of the proposed methods in Section 3. Section 4 concludes the paper.

\section{Moving least square - one-dimensional-integrated radial basis function networks}

A schematic outline of the MLS-1D-IRBFN method is depicted in Fig. 1. For brevity, 3-node support domains are presented here. Similar explanation is applicable in the case of 5-node support domains. On an $x$-grid line $[l]$, a global interpolant for the field variable at a grid point $x_{i}$ is sought as

$$
u\left(x_{i}\right)=\sum_{j=1}^{n} \phi_{j}\left(x_{i}\right) u_{j}\left(x_{i}\right),
$$

where $\left\{\phi_{j}\right\}_{j=1}^{n}$ is a set of the partition of unity functions constructed using MLS approximants, $u_{j}\left(x_{i}\right)$ is the nodal function value obtained from a local interpolant represented by $1 \mathrm{D}-\mathrm{IRBFNs}$, and $n$ is the number of nodes in the support domain of $x_{i}$. In (1), MLS approximants are presently based on linear polynomials, which are defined in terms of 1 and $x$. Relevant derivatives of $u$ at $x_{i}$ can be obtained by differentiating (1)

$$
\begin{aligned}
& \frac{\partial u\left(x_{i}\right)}{\partial x}=\sum_{j=1}^{n}\left(\frac{\partial \phi_{j}\left(x_{i}\right)}{\partial x} u_{j}\left(x_{i}\right)+\phi_{j}\left(x_{i}\right) \frac{\partial u_{j}\left(x_{i}\right)}{\partial x}\right) \\
& \frac{\partial^{2} u\left(x_{i}\right)}{\partial x^{2}}=\sum_{j=1}^{n}\left(\frac{\partial^{2} \phi_{j}\left(x_{i}\right)}{\partial x^{2}} u_{j}\left(x_{i}\right)+2 \frac{\partial \phi_{j}\left(x_{i}\right)}{\partial x} \frac{\partial u_{j}\left(x_{i}\right)}{\partial x}+\phi_{j}\left(x_{i}\right) \frac{\partial^{2} u_{j}\left(x_{i}\right)}{\partial x^{2}}\right),
\end{aligned}
$$

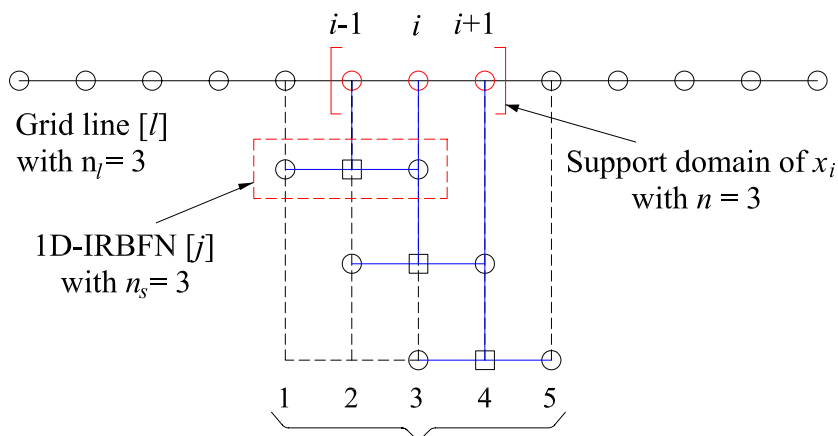

MLS-1D-IRBF network $[i]$ with $\mathrm{n}_{r}=5$

Figure 1: MLS-1D-IRBFN scheme, $\square$ a typical $[j]$ node. 
where the values $u_{j}\left(x_{i}\right), \partial u_{j}\left(x_{i}\right) / \partial x$ and $\partial^{2} u_{j}\left(x_{i}\right) / \partial x^{2}$ are calculated from 1DIRBFNs with $n_{s}$ nodes.

\subsection{One-dimensional IRBFN}

Consider a segment $[j]$ with $n_{s}$ nodes on an $x$-grid line $[l]$ as shown in Fig. 1 . The variation of the nodal function $u_{j}$ along this segment is sought in the IRBF form. The second-order derivative of $u_{j}$ is decomposed into RBFs; the RBF network is then integrated once and twice to obtain expressions for the first-order derivative of $u_{j}$ and the function $u_{j}$ itself as follows.

$$
\begin{aligned}
& \frac{\partial^{2} u_{j}(x)}{\partial x^{2}}=\sum_{k=1}^{n_{s}} w^{(k)} G^{(k)}(x), \\
& \frac{\partial u_{j}(x)}{\partial x}=\sum_{k=1}^{n_{s}} w^{(k)} H_{[1]}^{(k)}(x)+c_{1}, \\
& u_{j}(x)=\sum_{k=1}^{n_{s}} w^{(k)} H_{[0]}^{(k)}(x)+c_{1} x+c_{2},
\end{aligned}
$$

where $\left\{w^{(k)}\right\}_{k=1}^{n_{s}}$ are RBF weights to be determined; $\left\{G^{(k)}\right\}_{k=1}^{n_{s}}$ are known RBFs, e.g., for the case of multiquadrics $G^{(k)}(x)=\sqrt{\left(x-x^{(k)}\right)^{2}+a^{(k) 2}}, a^{(k)}$ - the RBF width; $H_{[1]}^{(k)}(x)=\int G^{(k)}(x) d x ; H_{[0]}^{(k)}(x)=\int H_{[1]}^{(k)}(x) d x$; and $c_{1}$ and $c_{2}$ are integration constants which are also unknown.

It is more convenient to work in the physical space than in the network-weight space. The RBF coefficients including two integration constants can be related to the physically meaningful nodal variable values. The second- and first-order derivatives of the variable $u$ are expressed in terms of nodal variable values as follows.

$$
\frac{\partial^{2} u(x)}{\partial x^{2}}=\bar{D}_{2 x} \hat{u}^{[j]}+k_{2 x}(x), \quad \frac{\partial u(x)}{\partial x}=\bar{D}_{1 x} \hat{u}^{[j]}+k_{1 x}(x),
$$

where $k_{1 x}$ and $k_{2 x}$ are scalars whose values depend on $x$ and boundary values; and $\bar{D}_{1 x}$ and $\bar{D}_{2 x}$ are known vectors of length $n_{s}$.

By application of equation (7) to $n_{s}$ nodes on the segment $[j]$, the second- and first-order derivatives of $u_{j}$ at node $x_{i}$ can be determined as

$$
\begin{aligned}
& \frac{\partial^{2} \hat{u}_{j}\left(x_{i}\right)}{\partial x^{2}}=\hat{\mathbf{M}}_{2 x(i d k,:)} \hat{u}^{[j]}+\hat{k}_{2 x(i d k)}, \\
& \frac{\partial \hat{u}_{j}\left(x_{i}\right)}{\partial x}=\hat{\mathbf{M}}_{1 x(i d k,:)} \hat{u}^{[j]}+\hat{k}_{1 x(i d k)},
\end{aligned}
$$

where $\hat{\mathbf{M}}_{1 x}$ and $\hat{\mathbf{M}}_{2 x}$ are known matrices of dimension $n_{s} \times n_{s} ; \hat{k}_{1 x}$ and $\hat{k}_{2 x}$ are known vectors of length $n_{s}$; and $i d k$ is the index number indicating the location of node $x_{i}$ over the local network $j$. 


\subsection{Incorporation of MLS and 1D-IRBFN}

By substituting equations (8) and (9) into equations (2) and (3), the second- and first-order derivatives of the variable $u\left(x_{i}\right)$ can be expressed as

$$
\frac{\partial u\left(x_{i}\right)}{\partial x}=\hat{\mathbf{d}}_{1 x}^{[i]} \hat{u}^{[i]}+k_{1 x}^{[i]}, \quad \frac{\partial^{2} u\left(x_{i}\right)}{\partial x^{2}}=\hat{\mathbf{d}}_{2 x}^{[i]} \hat{u}^{[i]}+k_{2 x}^{[i]}
$$

where $\hat{u}^{[i]}=\left(u^{(1)}, u^{(2)}, \ldots, u^{\left(n_{r}\right)}\right)^{T}, n_{r}$ is the number of nodes in the MLS-1DIRBF network $[i], k_{1 x}^{[i]}$ and $k_{2 x}^{[i]}$ are known scalars, and $\hat{\mathbf{d}}_{1 x}^{[i]}$ and $\hat{\mathbf{d}}_{2 x}^{[i]}$ are known vectors of length $n_{r}$.

The values of first- and second-order derivatives of $u$ with respect to $x$ at the nodal points on the grid line $[l]$ can be given by

$$
\frac{\partial \hat{u}_{i}}{\partial x}=\hat{\mathbf{D}}_{1 x}^{[l]} \hat{u}^{[l]}+\hat{k}_{1 x}^{[l]}, \quad \frac{\partial^{2} \hat{u}_{i}}{\partial x^{2}}=\hat{\mathbf{D}}_{2 x}^{[l]} \hat{u}^{[l]}+\hat{k}_{2 x}^{[l]}
$$

where $\hat{u}^{[l]}=\left(u^{(1)}, u^{(2)}, \ldots, u^{\left(n_{l}\right)}\right)^{T}, \hat{k}_{1 x(i)}^{[l]}$ and $\hat{k}_{2 x(i)}^{[l]}$ are known vectors of length $n_{l}, \hat{\mathbf{D}}_{1 x(i, i d i)}^{[l]}$ and $\hat{\mathbf{D}}_{2 x(i, i d i)}^{[l]}$ are known matrices of dimension $n_{l} \times n_{l}$, and $n_{l}$ is the number of nodes on the grid line $[l]$.

The values of first- and second-order derivatives of $u$ with respect to $x$ at the nodal points over the problem domain can be given by

$$
\frac{\partial \tilde{u}}{\partial x}=\tilde{\mathbf{D}}_{1 x} \tilde{u}+\tilde{k}_{1 x}, \quad \frac{\partial^{2} \tilde{u}}{\partial x^{2}}=\tilde{\mathbf{D}}_{2 x} \tilde{u}+\tilde{k}_{2 x},
$$

where $\tilde{u}=\left(u^{(1)}, u^{(2)}, \ldots, u^{\left(N_{i p}\right)}\right)^{T}, \tilde{\mathbf{D}}_{1 x}$ and $\tilde{\mathbf{D}}_{2 x}$ are known matrices of dimension $N_{i p} \times N_{i p} ; \tilde{k}_{1 x}$ and $\tilde{k}_{2 x}$ are known vectors of length $N_{i p}$; and $N_{i p}$ is the total number of interior nodal points.

Similarly, the values of the second- and first-order derivatives of $u$ with respect to $y$ at the nodal points over the problem domain can be given by

$$
\frac{\partial \tilde{u}}{\partial y}=\tilde{\mathbf{D}}_{1 y} \tilde{u}+\tilde{k}_{1 y}, \quad \frac{\partial^{2} \tilde{u}}{\partial y^{2}}=\tilde{\mathbf{D}}_{2 y} \tilde{u}+\tilde{k}_{2 y},
$$

\section{Numerical results}

The MLS-1D-IRBFN based on 5-node support domains and 5-node 1D-IRBFNs are applied to solve the following examples.

\subsection{Example 1: Two-dimensional diffusion equation}

This example is concerned with the following 2D diffusion equation

$$
\frac{\partial u}{\partial t}=\frac{\partial^{2} u}{\partial x^{2}}+\frac{\partial^{2} u}{\partial y^{2}}+f(x, y, t) \text { with } f(x, y, t)=\sin x \sin y(2 \sin t+\cos t),
$$


Table 1: Two-dimensional diffusion equation: comparison of relative error and CPU time between 1D-IRBFN and MLS-1D-IRBFN methods. The fully discrete scheme with standard $\theta$-scheme for temporal discretisation is employed. (1) 1D-IRBFN, (2) MLS-1D-IRBFN.

\begin{tabular}{|c|c|c|c|c|c|c|c|c|}
\hline \multirow[t]{2}{*}{ Grid } & \multirow[t]{2}{*}{$\Delta t$} & \multirow[t]{2}{*}{ Exact } & \multicolumn{2}{|c|}{ Numerical solution } & \multicolumn{2}{|c|}{ Relative error } & \multicolumn{2}{|c|}{ CPU time $(s)$} \\
\hline & & & (1) & (2) & (1) & (2) & (1) & (2) \\
\hline 11 & 1.0000 & -0.389450 & -0.387900 & -0.387891 & $3.98 \mathrm{E}-03$ & $4.00 \mathrm{E}-03$ & 0.02 & 0.19 \\
\hline 21 & 0.2500 & -0.389450 & -0.389404 & -0.389400 & 1.19E-04 & $1.30 \mathrm{E}-04$ & 0.16 & 0.78 \\
\hline 31 & 0.1111 & -0.389450 & -0.389441 & -0.389437 & $2.29 \mathrm{E}-05$ & $3.47 \mathrm{E}-05$ & 1.61 & 1.92 \\
\hline 41 & 0.0625 & -0.389450 & -0.389448 & -0.389443 & $7.23 \mathrm{E}-06$ & $1.93 \mathrm{E}-05$ & 8.36 & 3.76 \\
\hline 51 & 0.0400 & -0.389450 & -0.389449 & -0.389444 & 2.95E-06 & $1.51 \mathrm{E}-05$ & 40.97 & 6.48 \\
\hline 61 & 0.0278 & -0.389450 & -0.389450 & -0.389445 & $1.42 \mathrm{E}-06$ & $1.36 \mathrm{E}-05$ & 127.39 & 10.47 \\
\hline
\end{tabular}

Table 2: Two-dimensional diffusion equation: MLS-1D-IRBFN solutions at the interior point $x=0.8, y=0.8$ in comparison with those of BEM [9] and 1D-IRBFN methods with the same time step $\Delta t=0.25$. The same grid of $21 \times 21$ is employed for 1D-IRBFN and MLS-1D-IRBF methods, while a much finer mesh is used for BEM. The fully discrete scheme with standard $\theta$-scheme for temporal discretisation is employed. (1) 1DIRBFN, (2) MLS-1D-IRBFN.

\begin{tabular}{|c|c|c|c|c|c|c|c|}
\hline \multirow[t]{2}{*}{$T$} & \multirow[t]{2}{*}{ Exact } & \multicolumn{3}{|c|}{ Numerical solution } & \multicolumn{3}{|c|}{ Relative error } \\
\hline & & BEM & (1) & (2) & BEM & (1) & (2) \\
\hline 0.25 & 0.127314 & 0.126800 & 0.127206 & 0.127205 & $4.04 \mathrm{E}-03$ & $8.45 \mathrm{E}-04$ & $8.55 \mathrm{E}-04$ \\
\hline 0.50 & 0.246712 & 0.245300 & 0.246679 & 0.246676 & $5.72 \mathrm{E}-03$ & $1.34 \mathrm{E}-04$ & $1.45 \mathrm{E}-04$ \\
\hline 0.75 & 0.350771 & 0.348500 & 0.350701 & 0.350697 & $6.47 \mathrm{E}-03$ & $2.00 \mathrm{E}-04$ & $2.12 \mathrm{E}-04$ \\
\hline 1.00 & 0.433021 & 0.430000 & 0.432997 & 0.432992 & $6.98 \mathrm{E}-03$ & $5.48 \mathrm{E}-05$ & $6.62 \mathrm{E}-05$ \\
\hline 1.25 & 0.488347 & 0.484800 & 0.488313 & 0.488308 & $7.26 \mathrm{E}-03$ & $6.98 \mathrm{E}-05$ & $8.12 \mathrm{E}-05$ \\
\hline 1.50 & 0.513311 & 0.509400 & 0.513313 & 0.513307 & 7.62E-03 & 4.23E-06 & $7.36 \mathrm{E}-06$ \\
\hline 1.75 & 0.506359 & 0.502400 & 0.506363 & 0.506357 & $7.82 \mathrm{E}-03$ & 7.23E-06 & 4.44E-06 \\
\hline 2.00 & 0.467924 & 0.464100 & 0.467956 & 0.467951 & $8.17 \mathrm{E}-03$ & $6.89 \mathrm{E}-05$ & $5.70 \mathrm{E}-05$ \\
\hline 2.25 & 0.400396 & 0.397000 & 0.400433 & 0.400429 & $8.48 \mathrm{E}-03$ & $9.27 \mathrm{E}-05$ & 8.07E-05 \\
\hline 2.50 & 0.307974 & 0.305200 & 0.308031 & 0.308027 & $9.01 \mathrm{E}-03$ & $1.85 \mathrm{E}-04$ & $1.73 \mathrm{E}-04$ \\
\hline 2.75 & 0.196403 & 0.194400 & 0.196462 & 0.196460 & $1.02 \mathrm{E}-02$ & $3.03 \mathrm{E}-04$ & $2.90 \mathrm{E}-04$ \\
\hline 3.00 & 0.072620 & 0.071500 & 0.072690 & 0.072689 & $1.54 \mathrm{E}-02$ & $9.54 \mathrm{E}-04$ & $9.39 \mathrm{E}-04$ \\
\hline 3.25 & -0.055677 & -0.055800 & -0.055611 & -0.055611 & $2.21 \mathrm{E}-03$ & $1.19 \mathrm{E}-03$ & $1.20 \mathrm{E}-03$ \\
\hline 3.50 & -0.180513 & -0.179700 & -0.180447 & -0.180446 & $4.50 \mathrm{E}-03$ & $3.63 \mathrm{E}-04$ & $3.74 \mathrm{E}-04$ \\
\hline 3.75 & -0.294125 & -0.292300 & -0.294070 & -0.294066 & $6.21 \mathrm{E}-03$ & $1.90 \mathrm{E}-04$ & $2.01 \mathrm{E}-04$ \\
\hline 4.00 & -0.389450 & -0.386800 & -0.389404 & -0.389400 & $6.81 \mathrm{E}-03$ & $1.19 \mathrm{E}-04$ & $1.30 \mathrm{E}-04$ \\
\hline
\end{tabular}


Table 3: Two-dimensional diffusion equation: MLS-1D-IRBFN solutions at the interior point $x=0.8, y=0.8$ in comparison with those of BEM [9] and $1 \mathrm{D}-\mathrm{IRBFN}$ methods with the same time step $\Delta t=0.25$. The same grid of $21 \times 21$ is employed for 1D-IRBFN and MLS-1D-IRBF methods, while a much finer mesh is used for BEM. The semi-discrete scheme with fourth-order Runge-Kutta method for temporal discretisation is employed. (1) 1D-IRBFN, (2) MLS-1D-IRBFN.

\begin{tabular}{|c|c|c|c|c|c|c|c|}
\hline \multirow[t]{2}{*}{$T$} & \multirow[t]{2}{*}{ Exact } & \multicolumn{3}{|c|}{ Numerical solution } & \multicolumn{3}{|c|}{ Relative error } \\
\hline & & BEM & (1) & (2) & BEM & (1) & (2) \\
\hline 0.25 & 0.127314 & 0.126800 & 0.127315 & 0.127314 & $4.04 \mathrm{E}-03$ & $6.45 \mathrm{E}-06$ & $1.64 \mathrm{E}-06$ \\
\hline 0.50 & 0.246712 & 0.245300 & 0.246714 & 0.246712 & $5.72 \mathrm{E}-03$ & 7.33E-06 & $2.14 \mathrm{E}-06$ \\
\hline 0.75 & 0.350771 & 0.348500 & 0.350774 & 0.350770 & $6.47 \mathrm{E}-03$ & $7.65 \mathrm{E}-06$ & $2.32 \mathrm{E}-06$ \\
\hline 1.00 & 0.433021 & 0.430000 & 0.433024 & 0.433020 & $6.98 \mathrm{E}-03$ & 7.84E-06 & $2.43 \mathrm{E}-06$ \\
\hline 1.25 & 0.488347 & 0.484800 & 0.488351 & 0.488346 & $7.26 \mathrm{E}-03$ & 7.97E-06 & $2.50 \mathrm{E}-06$ \\
\hline 1.50 & 0.513311 & 0.509400 & 0.513315 & 0.513309 & $7.62 \mathrm{E}-03$ & $8.08 \mathrm{E}-06$ & $2.57 \mathrm{E}-06$ \\
\hline 1.75 & 0.506359 & 0.502400 & 0.506363 & 0.506358 & $7.82 \mathrm{E}-03$ & 8.19E-06 & $2.63 \mathrm{E}-06$ \\
\hline 2.00 & 0.467924 & 0.464100 & 0.467928 & 0.467923 & $8.17 \mathrm{E}-03$ & 8.31E-06 & $2.69 \mathrm{E}-06$ \\
\hline 2.25 & 0.400396 & 0.397000 & 0.400400 & 0.400395 & $8.48 \mathrm{E}-03$ & $8.45 \mathrm{E}-06$ & $2.78 \mathrm{E}-06$ \\
\hline 2.50 & 0.307974 & 0.305200 & 0.307976 & 0.307973 & $9.01 \mathrm{E}-03$ & $8.68 \mathrm{E}-06$ & $2.91 \mathrm{E}-06$ \\
\hline 2.75 & 0.196403 & 0.194400 & 0.196404 & 0.196402 & $1.02 \mathrm{E}-02$ & $9.14 \mathrm{E}-06$ & $3.17 \mathrm{E}-06$ \\
\hline 3.00 & 0.072620 & 0.071500 & 0.072621 & 0.072620 & $1.54 \mathrm{E}-02$ & $1.11 \mathrm{E}-05$ & $4.28 \mathrm{E}-06$ \\
\hline 3.25 & -0.055677 & -0.055800 & -0.055677 & -0.055677 & $2.21 \mathrm{E}-03$ & 4.19E-06 & $3.58 \mathrm{E}-07$ \\
\hline 3.50 & -0.180513 & -0.179700 & -0.180514 & -0.180513 & $4.50 \mathrm{E}-03$ & $6.97 \mathrm{E}-06$ & $1.94 \mathrm{E}-06$ \\
\hline 3.75 & -0.294125 & -0.292300 & -0.294128 & -0.294125 & $6.21 \mathrm{E}-03$ & $7.50 \mathrm{E}-06$ & $2.24 \mathrm{E}-06$ \\
\hline 4.00 & -0.389450 & -0.386800 & -0.389453 & -0.389449 & $6.81 \mathrm{E}-03$ & 7.74E-06 & $2.37 \mathrm{E}-06$ \\
\hline
\end{tabular}

defined on a square domain $0<x, y<1, t>0$ and subject to Dirichlet boundary conditions. The boundary and initial conditions can be derived from the analytical solution $u_{E}=\sin x \sin y \sin t$.

In this example, the time step $\Delta t$ is taken based on the parameter $d=$ $\kappa \Delta t / \Delta x^{2}$, where $\kappa$ is the diffusion coefficient, presently $\kappa=1$. The parameter $d$ is the ratio of time step $\Delta t$ to the characteristic diffusion time $\Delta x^{2} / \kappa$, which is roughly the time required for a disturbance to be transmitted by diffusion over a distance $\Delta x$ [8]. The parameter $d$ is here chosen to be 100 .

The grid convergence study and CPU-time requirement for both 1D-IRBFN and MLS-1D-IRBFN based on the fully discrete framework with standard $\theta$ scheme for temporal discretisation are presented in Table 1. It can be seen that the numerical solutions for both methods are converging well from the coarse mesh to the fine mesh. MLS-1D-IRBFN yields the same order accuracy as that of 1D-IRBFN, and offers a significant improvement in terms of efficiency when dealing with fine meshes. Table 2 presents the numerical solutions of 1D-IRBFN and MLS-1D-IRBFN methods at the interior point $x=0.8, y=0.8$ in comparison 
Table 4: One-dimensional advection-diffusion equation: MLS-1D-IRBFN solutions at time $t=1$ in comparison with those of DRBFN [3] and 1DIRBFN methods with the same grid of 17 and $\Delta t=0.01$. (1) $1 \mathrm{D}-\mathrm{IRBFN}$, (2) MLS-1D-IRBFN.

\begin{tabular}{|c|c|c|c|c|c|c|c|}
\hline \multirow[t]{2}{*}{$x$} & \multirow[t]{2}{*}{ Exact } & \multicolumn{3}{|c|}{ Numerical solution } & \multicolumn{3}{|c|}{ Absolute error } \\
\hline & & DRBFN & (1) & (2) & DRBFN & (1) & (2) \\
\hline 0.0625 & 0.998874 & - & 0.998880 & 0.998882 & - & $6.51 \mathrm{E}-06$ & $8.44 \mathrm{E}-06$ \\
\hline 0.1250 & 0.902800 & - & 0.902804 & 0.902810 & - & $4.18 \mathrm{E}-06$ & $9.95 \mathrm{E}-06$ \\
\hline 0.1875 & 0.815967 & - & 0.815970 & 0.815982 & - & $2.76 \mathrm{E}-06$ & $1.51 \mathrm{E}-05$ \\
\hline 0.2500 & 0.737486 & 0.737486 & 0.737487 & 0.737506 & $9.89 \mathrm{E}-03$ & $1.15 \mathrm{E}-06$ & $1.98 \mathrm{E}-05$ \\
\hline 0.3125 & 0.666553 & 0.666554 & 0.666553 & 0.666576 & $8.45 \mathrm{E}-03$ & $3.45 \mathrm{E}-07$ & $2.27 \mathrm{E}-05$ \\
\hline 0.3750 & 0.602443 & 0.602444 & 0.602441 & 0.602467 & $6.37 \mathrm{E}-03$ & $1.88 \mathrm{E}-06$ & $2.41 \mathrm{E}-05$ \\
\hline 0.4375 & 0.544499 & - & 0.544495 & 0.544523 & - & 3.44E-06 & $2.43 \mathrm{E}-05$ \\
\hline 0.5000 & 0.492128 & 0.492129 & 0.492123 & 0.492151 & $1.78 \mathrm{E}-03$ & $5.05 \mathrm{E}-06$ & $2.36 \mathrm{E}-05$ \\
\hline 0.5625 & 0.444794 & - & 0.444787 & 0.444816 & - & $6.73 \mathrm{E}-06$ & $2.22 \mathrm{E}-05$ \\
\hline 0.6250 & 0.402013 & 0.402014 & 0.402004 & 0.402033 & $1.82 \mathrm{E}-03$ & $8.48 \mathrm{E}-06$ & $2.02 \mathrm{E}-05$ \\
\hline 0.6875 & 0.363346 & - & 0.363336 & 0.363364 & - & $1.03 \mathrm{E}-05$ & $1.77 \mathrm{E}-05$ \\
\hline 0.7500 & 0.328399 & 0.328400 & 0.328387 & 0.328414 & $3.66 \mathrm{E}-03$ & $1.21 \mathrm{E}-05$ & $1.48 \mathrm{E}-05$ \\
\hline 0.8125 & 0.296813 & - & 0.296799 & 0.296825 & - & $1.42 \mathrm{E}-05$ & $1.15 \mathrm{E}-05$ \\
\hline 0.8750 & 0.268265 & 0.268266 & 0.268249 & 0.268272 & 4.01E-03 & $1.57 \mathrm{E}-05$ & $7.34 \mathrm{E}-06$ \\
\hline 0.9375 & 0.242463 & - & 0.242444 & 0.242465 & - & $1.93 \mathrm{E}-05$ & $1.85 \mathrm{E}-06$ \\
\hline
\end{tabular}

with those of BEM, which were published in [9] using the first-order finite difference approximation for the time derivative and boundary element method for spatial discretisation. The corresponding results of 1D-IRBFN and MLS-1DIRBFN based on the semi-discrete framework with fourth-order Runge-Kutta method for temporal discretisation are described in Table 3. The same grid of $21 \times 21$ is used for 1D-IRBFN and MLS-1D-IRBF methods, while a much finer mesh was used for BEM. It can be seen in these tables that the results of MLS-1DIRBFN and 1D-IRBFN methods are slightly different and both are more accurate than that of BEM.

\subsection{Example 2: One-dimensional advection-diffusion equation}

The present method is here applied to solve the $1 \mathrm{D}$ advection-diffusion equation $\partial u(x, t) / \partial t=\kappa \partial^{2} u(x, t) / \partial x^{2}+\nu \partial u(x, t) / \partial x$, defined on a domain $0<x<1$, $t>0$ and subject to boundary and initial conditions $u(0, t)=a e^{b t}, t>0$, $u(1, t)=a e^{b t-c}, t>0$, and $u(x, 0)=a e^{-c x}, 0 \leq x \leq 1$. The problem has an analytical solution $u_{E}=a e^{b t-c x}$, where $c=\left(\nu+\sqrt{\nu^{2}+4 \kappa b}\right) /(2 \kappa)>0$, $\kappa=0.1, b=0.1, a=1.0$, and $\nu=0.1$.

Table 4 presents the MLS-1D-IRBFN numerical solutions of the $1 \mathrm{D}$ advectiondiffusion equation at time $t=1 s$ in comparison with those of DRBFN [3] and 1DIRBFN methods. The DRBFN result was obtained using implicit Crank-Nicholson 
Table 5: Forced vibration of simply supported beam: comparison of deflection $w$ at time $t=1$ s. (1) $1 \mathrm{D}-\mathrm{IRBFN}$, (2) MLS-1D-IRBFN.

\begin{tabular}{llccccc}
\hline$x(\mathrm{~cm})$ & \multirow{2}{*}{ Exact } & \multicolumn{2}{c}{ Numerical solution } & & \multicolumn{2}{c}{ Relative error } \\
\cline { 3 - 4 } \cline { 7 - 7 } & & $(1)$ & $(2)$ & & $(1)$ & $(2)$ \\
\hline 5 & -0.009151 & -0.009140 & -0.009143 & & $1.24 \mathrm{E}-03$ & $9.04 \mathrm{E}-04$ \\
10 & -0.016306 & -0.016289 & -0.016293 & & $1.05 \mathrm{E}-03$ & $7.72 \mathrm{E}-04$ \\
15 & -0.019916 & -0.019891 & -0.019896 & & $1.24 \mathrm{E}-03$ & $9.84 \mathrm{E}-04$ \\
20 & -0.020138 & -0.020109 & -0.020114 & & $1.45 \mathrm{E}-03$ & $1.21 \mathrm{E}-03$ \\
25 & -0.017585 & -0.017560 & -0.017564 & & $1.44 \mathrm{E}-03$ & $1.21 \mathrm{E}-03$ \\
30 & -0.012916 & -0.012898 & -0.012901 & & $1.44 \mathrm{E}-03$ & $1.20 \mathrm{E}-03$ \\
35 & -0.006820 & -0.006809 & -0.006810 & & $1.70 \mathrm{E}-03$ & $1.45 \mathrm{E}-03$ \\
\hline
\end{tabular}

Table 6: Forced vibration of simply supported beam: comparison of velocity $v$ at time $t=1$ s. (1) $1 \mathrm{D}-\mathrm{IRBFN}$, (2) MLS-1D-IRBFN.

\begin{tabular}{lcccccc}
\hline$x(\mathrm{~cm})$ & Exact & \multicolumn{2}{c}{ Numerical solution } & & \multicolumn{2}{c}{ Relative error } \\
\cline { 3 - 4 } \cline { 6 - 7 } & & $(1)$ & $(2)$ & & $(1)$ & $(2)$ \\
\hline 5 & 1.558434 & 1.560281 & 1.561015 & & $1.19 \mathrm{E}-03$ & $1.66 \mathrm{E}-03$ \\
10 & 2.776816 & 2.779817 & 2.780941 & & $1.08 \mathrm{E}-03$ & $1.49 \mathrm{E}-03$ \\
15 & 3.391601 & 3.394314 & 3.395441 & & $8.00 \mathrm{E}-04$ & $1.13 \mathrm{E}-03$ \\
20 & 3.429398 & 3.430837 & 3.431784 & & $4.20 \mathrm{E}-04$ & $6.96 \mathrm{E}-04$ \\
25 & 2.994631 & 2.995018 & 2.995757 & & $1.29 \mathrm{E}-04$ & $3.76 \mathrm{E}-04$ \\
30 & 2.199611 & 2.199324 & 2.199909 & & $1.31 \mathrm{E}-04$ & $1.35 \mathrm{E}-04$ \\
35 & 1.161464 & 1.160977 & 1.161331 & & $4.20 \mathrm{E}-04$ & $1.15 \mathrm{E}-04$ \\
\hline
\end{tabular}

Thin Plate Spline scheme. For comparison purposes, 1D-IRBFN and MLS-1DIRBFN are implemented based on the fully discrete scheme with standard $\theta$ scheme for temporal discretisation, and using the same grid and time step as those in [3]. It can be seen that the MLS-1D-IRBFN result is more accurate than that DRBFN, but slightly less accurate than that of $1 \mathrm{D}-\mathrm{IRBFN}$. 


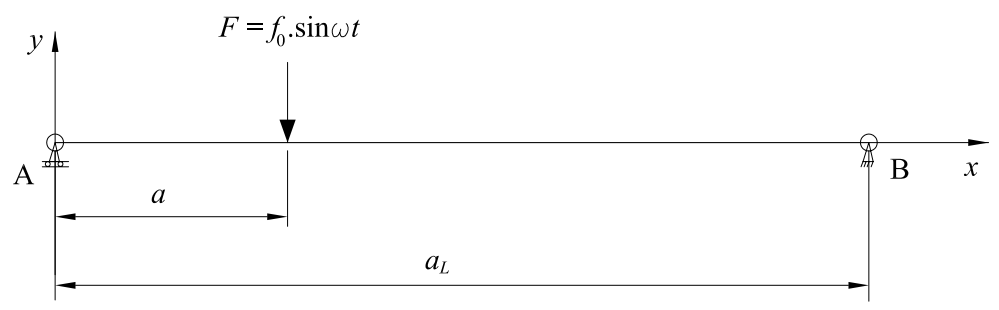

Figure 2: Forced vibration of a simply supported beam.
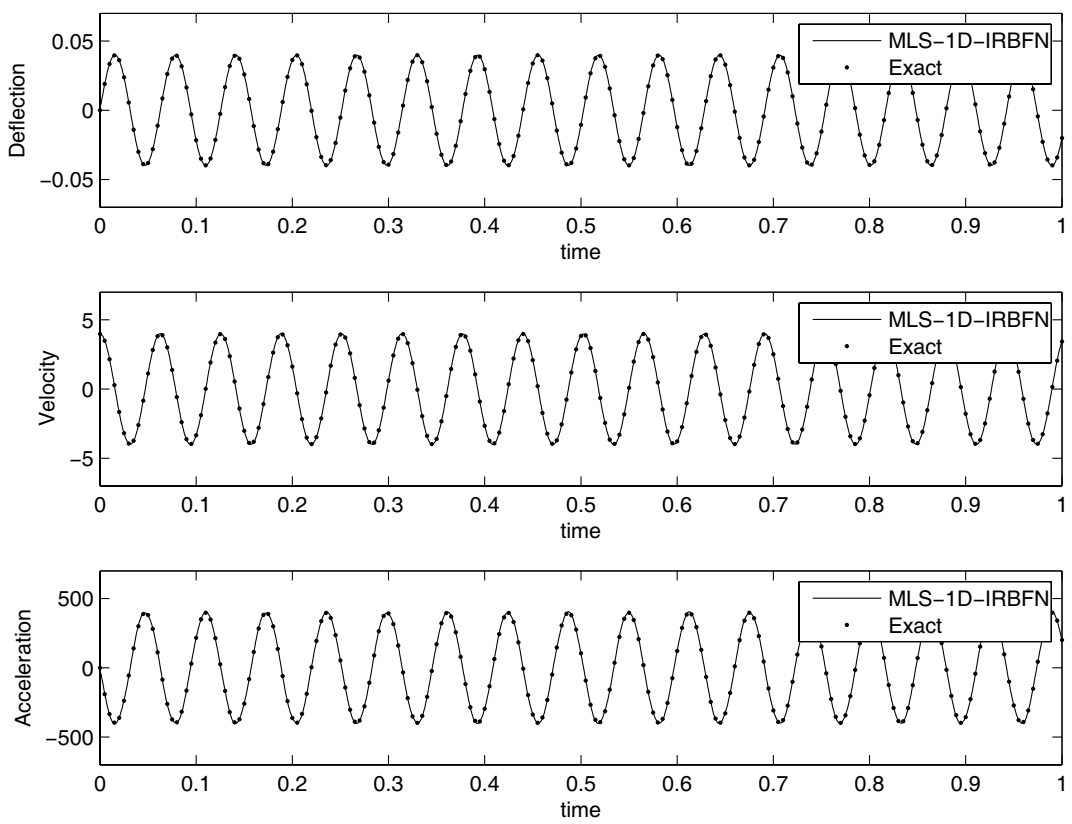

Figure 3: Steady state response of the mid-point of a simply supported beam, using a grid of 81 and $\Delta t=10^{-3}$.

\subsection{Example 3: Forced vibration of a beam}

This example deals with the dynamic behaviour of a simply supported beam subject to a harmonic external force $F(t)=f_{0} \sin \omega t$ applied at $x=a$, as shown in Fig. 2 (where $f_{0}=100 \mathrm{~N}, \omega=100 \mathrm{rad} / \mathrm{s}$ and $a=10 \mathrm{~cm}$ ). The problem geometry and material parameters of the beam used here are: the length of the beam $a_{L}=40 \mathrm{~cm}$, the cross-section area $A=1 \mathrm{~cm}^{2}$, the moment of inertia $I=0.0833 \mathrm{~cm}^{4}$, Young's modulus $E=300 \mathrm{GPa}$ and material density $\rho=732.4 \mathrm{~kg} / \mathrm{m}^{3}$. The equation of motion for forced lateral vibration of a beam is given by $E I \partial^{4} w / \partial x^{4}+\rho A \partial^{2} w / \partial t^{2}=f(x, t)$. The boundary and initial 
conditions for the simply supported beam can be described as $w=0, \frac{\partial^{2} w}{\partial x^{2}}=0$, at $x=0, x=a_{L} ; w=0, \frac{\partial w}{\partial t}=v_{0}$, at $t=0$. An analytical solution to this problem can be found in [10].

Tables 5 and 6 present numerical results of the deflection and velocity of the simply supported beam at time $t=1 s$, using a grid of 81 and time step of $10^{-3}$. The fully discrete scheme with Newmark method for temporal discretisation is employed here. It can be seen that MLS-1D-IRBFN yields more accurate result for deflection than 1D-IRBFN, while 1D-IRBFN produces more accurate result for velocity than MLS-1D-IRBFN. Fig. 3 shows the steady-state responses of the forced vibration system using MLS-1D-IRBFN in comparison with the analytical solutions. The numerical results are in good agreement with the analytical solutions as shown in this figure.

\section{Conclusion}

A moving least square - one-dimensional-integrated radial basis function networks approach based on the semi-discrete and fully discrete frameworks is developed for time-dependent problems. Spatial discretisation is carried out using MLS-1DIRBFN and Cartesian grids, while the time derivatives are discretised using the high order schemes (e.g. standard $\theta$, fourth-order Runge-Kutta). The numerical results obtained show that the proposed methods yield a high convergence order of accuracy as that of 1D-IRBFN, while requires less computational effort. The present methods have a promising capability to solve unsteady incompressible viscous flow problems.

\section{Acknowledgement}

D. Ngo-Cong is supported by a University of Southern Queensland Postgraduate Research Scholarship.

\section{References}

[1] Kansa, E.J., Multiquadrics - A Scattered Data Approximation Scheme with Applications to Computational Fluid-Dynamics - II: Solutions to parabolic, Hyperbolic and Elliptic Partial Differential Equations. Computers \& Mathematics with Applications, 19(8/9), pp. 147-61, 1990.

[2] Sharan M., Kansa E.J. \& Gupta S., Application of multiquadric method for numerical solution of elliptic partial differential equations. Applied Mathematical Computations, 84(2-3), pp. 275-302, 1997.

[3] Zerroukat M., Djidjeli K. \& Charafi A., Explicit and implicit meshless methods for linear advection-diffusion-type partial differential equations. International Journal for Numerical Methods in Engineering, 48, pp. 19-35, 2000. 
[4] Ye T., Mittal R., Udaykumar H.S. \& Shyy W., An accurate Cartesian grid method for viscous incompressible flows with complex immersed boundaries. Journal of Computational Physics, 156, pp. 209-240, 1999.

[5] Mai-Duy N. \& Tanner R.I., A Collocation Method based on OneDimensional RBF Interpolation Scheme for Solving PDEs. International Journal of Numerical Methods for Heat \& Fluid Flow, 17(2), pp. 165-186, 2007.

[6] Le-Cao K., Mai-Duy N. \& Tran-Cong T., An effective integrated-RBFN Cartesian-grid discretization for the stream function-vorticity-temperature formulation in nonrectangular domains. Numerical Heat Transfer, Part B, 55, pp. 480-502, 2009.

[7] Ngo-Cong D., Mai-Duy N., Karunasena W. \& Tran-Cong T., Free vibration analysis of laminated composite plates based on FSDT using onedimensional IRBFN method. Computers \& Structures, 89, pp. 1-13, 2011.

[8] Ferziger J.H. \& Peric M., Computational Methods for Fluid Dynamics, 3rd edition, Springer: Berlin, pp. 143-144, 2002.

[9] Ingber M. \& Phan-Thien N., A boundary element approach for parabolic differential equations using a class of particular solutions. Applied Mathematical Modelling, 16(3), pp. 124-132, 1992.

[10] Rao S.S., Mechanical vibrations, 4th edition, Pearson Prentice Hall: New Jersey, pp. 619-620, 2004. 